Usages du français et pratiques d'enseignement en Europe balkanique, centrale et orientale - Grèce,

Serbie, Bulgarie, Moldavie, Hongrie, Allemagne, Russie - XVIIle - XXe siècles

\title{
Le premier manuel de français publié en Grèce
}

\author{
Jean Antoine Caravolas
}

\section{(2) OpenEdition Journals}

\section{Édition électronique}

URL : https://journals.openedition.org/dhfles/4155

DOI : $10.4000 /$ dhfles.4155

ISSN : 2221-4038

\section{Éditeur}

Société Internationale pour l'Histoire du Français Langue Étrangère ou Seconde

\section{Édition imprimée}

Date de publication : 1 juin 2015

Pagination : 63-78

ISSN : 0992-7654

Référence électronique

Jean Antoine Caravolas, "Le premier manuel de français publié en Grèce », Documents pour l'histoire du français langue étrangère ou seconde [En ligne], 54 | 2015, mis en ligne le 01 janvier 2018, consulté le 29 mars 2023. URL : http://journals.openedition.org/dhfles/4155 ; DOl : https://doi.org/10.4000/ dhfles. 4155

Ce document a été généré automatiquement le 29 mars 2023. 


\title{
Le premier manuel de français publié en Grèce
}

\author{
Jean Antoine Caravolas
}

1 Le 3 février 1830, après quatre siècles d'occupation turque (1453-1821) et huit ans de révolution armée (1821-1828), les plénipotentiaires de la Russie, de la France et de la Grande-Bretagne, réunis à Londres signaient, le protocole relatif à l'indépendance de la Grèce. Le nouvel état comptait environ 700000 habitants et ne comprenait que le Péloponnèse, une partie de la Grèce centrale, l'île Eubée et les Cyclades. Son gouvernement devait être monarchique et héréditaire. Plus tôt cependant, en avril 1827, l'Assemblée nationale des Grecs révoltés avait approuvé la constitution du pays et désigné le premier gouverneur du futur état indépendant le comte Ioannis Capodistrias. Par leur choix les législateurs espéraient que cet aristocrate originaire de l'île de Corfou, médecin diplômé de Padoue et ancien ministre des affaires étrangères du tsar Alexandre (1815-1822), mettrait, par son prestige et son expérience, fin aux querelles entre les clans et réaliserait les réformes prévues par la constitution.

2 Dès son arrivée en Grèce, le 8 janvier 1828, Capodistrias s'appliqua à la création d'un système national d'éducation. La tâche s'annonçait extrêmement ardue, car, pendant la révolution, la plupart des écoles avaient été fermées ou ruinées, le matériel didactique détruit et les maîtres d'école dispersés ou tués. Néanmoins, trois ans plus tard, en 1831,

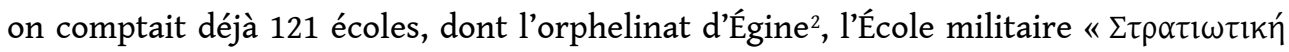

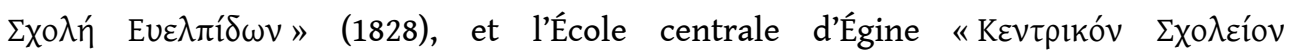
Alyívns »(1829) (Chadzistefanides $1986: 46)$.

\section{L'enseignement du français}

3 Dans la dédicace Aux Grecs libres de la mer Ionienne de sa traduction française des Caractères de Théophraste (1799), Coray, le père des Lumières grecques, écrit à ses compatriotes que, s'ils souhaitent vraiment devenir les «émules » ou les « rivaux » de leurs "glorieux ancêtres ", ils doivent apprendre, entre autres, le grec ancien (langue des dieux) et le français (langue de la raison et de la philosophie) (cité dans Nicolaïdis 1992). 
La connaissance du français était jugée indispensable pour les politiciens, pour les professeurs et surtout pour les militaires, l'armée régulière grecque ayant été formée, équipée et longtemps dirigée par des officiers français ${ }^{3}$.

Outre le contrôle sur l'armée, la France exerçait également une grande influence sur la vie politique du pays, longtemps dominée par le parti français de Jean Colettis (1773-1847)4. Il n'est donc pas surprenant que le français ait été enseigné à l'École militaire et à l'École centrale, dès leur fondation.

\section{L'enseignement du français à l'École Centrale}

5 L'École centrale avait pour mission de former des instituteurs pour les écoles mutuelles et les écoles régulières. Le premier professeur de français à l'École centrale était Jean Venthylos (1804-1854), un pédagogue formé en Allemagne. Après quelques semaines toutefois, il donna sa démission ${ }^{5}$. On nomma alors pour enseigner le français Anastasios Herculides, un personnage sur lequel nous possédons très peu d'informations. Il arriva à Égine le 28 mai 1829, au moment où la lutte entre les amis et les ennemis de Capodistrias atteignait son paroxysme. Il semble que, contrairement à son prédécesseur et nombre de ses collègues ${ }^{6}$ et étudiants, il ait été plutôt favorable au Gouverneur.

6 La tension au pays montait de jour en jour. Au début de janvier 1831 les étudiants de l'École centrale se révoltèrent. L'École comptait alors environ 300 élèves, âgés en moyenne de vingt ans et venus de différentes régions de la Grèce. Les étudiants se plaignaient de n'avoir pas trouvé à Égine des maîtres de qualité ni reçu tous les cours annoncés au programme d'études. Ils refusaient les solutions provisoires, exigeaient le congédiement immédiat du professeur de mathématiques qu'ils accusaient d'incompétence, ainsi que celui de Herculides, à qui ils reprochaient de traduire « les mots français par des mots turcs ", de ne permettre à personne d'entrer dans la salle de classe après le son de la cloche et de vouloir diviser les élèves en groupes selon leurs connaissances.

7 Le 6 janvier 1831, Capodistrias informé sur la mutinerie, ordonna la fermeture de l'établissement et la punition des meneurs. Les leçons furent alors dispensées chez les élèves qui voulaient étudier. Pas pour longtemps. Le 20 janvier ils refusèrent tous unanimement de suivre les cours d'Herculides, mais deux jours plus tard, les étudiants présentèrent au commissaire leurs excuses et les cours reprirent plus ou moins normalement. (Dimaras 1973: 32) ${ }^{7}$. Herculides continua à dispenser ses cours et prépara même pour ses élèves un manuel de français qu'il fit imprimer à l'imprimerie nationale d'Égine. Soudain, le 9 octobre 1831, le gouverneur est assassiné. Le pays sombra alors dans l'anarchie et la plupart des écoles y compris l'École centrale furent fermées.

\section{La fin de l'École centrale}

Les puissances protectrices imposent alors Othon $1^{\mathrm{er}}$ (1815-1867), prince bavarois de 17 ans, comme premier roi de Grèce. Il arrive avec sa suite le 30 janvier 1833 et s'installe à Athènes qui, en 1834, devient la capitale du royaume. Une nouvelle page de l'histoire de la Grèce moderne commence. 
9 La situation dans le domaine de l'éducation change aussi. Le gouvernement ouvrit de nombreuses nouvelles écoles, surtout secondaires. La langue d'enseignement et des manuels de toutes les classes était le grec ancien, que les élèves ne comprenaient pas. En 1836, les écoles du niveau secondaire sont divisées, selon le modèle allemand

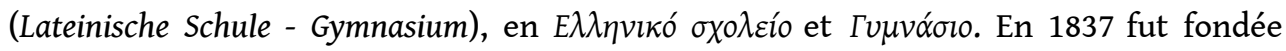
l'Université d'Athènes.

\section{Le manuel de français d'Herculides}

10 L'étude du français à l'école secondaire, occupe dans les nouveaux programmes d'études, une place importante, surtout dans les classes inférieures ${ }^{8}$. Mais les manuels manquaient toujours. Le premier à être composé par un auteur grec et publié par un

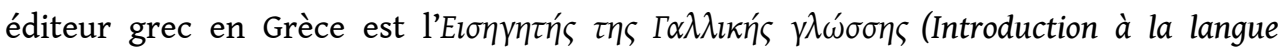

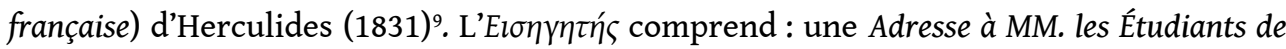
l'École centrale d'Égine (p. $\alpha, \beta)$, où l'auteur explique en français son but et exprime le vœu que son manuel éveille en eux "le goût pour l'étude de cette belle langue »; L'Avertissement ( $\mathrm{p}, \gamma-\zeta)$ où il expose brièvement ${ }^{10}$ la procédure adoptée lors de la rédaction du manuel et décrit la manière dont maîtres et élèves devaient s'en servir.

11 L'auteur croyait qu'il y avait deux objectifs dans l'étude d'une langue étrangère : comprendre les auteurs et apprendre à parler et à écrire la langue correctement. Il était convaincu que c'est par la méthode de lecture que l'on les atteint le plus facilement: «La lecture qui réunit l'avantage d'amuser et d'instruire ressemble à un fruit délicieux et nourrissant à la fois ». Cette phrase, extraite des Élémens de littérature (1787) de Marmontel, imprimée sur la page de titre de son manuel, résume bien la conception de l'apprentissage des langues d'Hercoulides.

12 L'histoire de la didactique des langues connaît plusieurs variantes de la méthode de lecture (Caravolas 2000). Une des plus répandues était la méthode d'« enseignement universel » de Jean Joseph Jacotot (1770-1840) ${ }^{11}$. Herculides la considérait comme la plus rapide pour la culture de la mémoire et du goût des jeunes (ibid. : $\varepsilon$ ). Elle suppose toutefois un accès facile à un grand nombre de livres de genres différents. Les livres étrangers étant, les premières années de l'histoire de l'État grec, introuvables, Herculides décida d' :

obvier à l'embarras où vous [les étudiants] jetaient dès le début de l'enseignement de la langue française dans notre école, les difficultés qu'il y avait à vous procurer des livres élémentaires; suppléer par un seul volume peu dispendieux, à tant de livres qu'exigerait l'étude d'une langue d'une langue aussi cultivée qu'est la langue française; vous donner en même temps un faible échantillon de sa belle et vaste littérature; en aplanir l'intelligence par quelques notes et par l'explication des mots contenus dans le texte : tel a été le but que s'est proposé l'éditeur en donnant au jour ce petit ouvrage. (Herculides $1831:$ a)

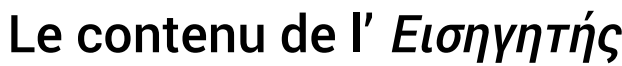

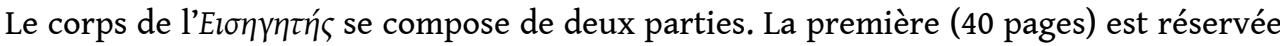
aux débutants. Elle enseigne les lettres, leur prononciation (p. 1-11) et leur écriture (la calligraphie, p. 12-14); présente des textes de lecture faciles (quelques brèves 
anecdotes (p. 12-14 ${ }^{12}$; deux dialogues ${ }^{13}$ avec le vocabulaire expliqué en grec, à la fin du texte et des brefs récits (p. 32-39).

La deuxième partie (p. 41-61) du manuel s'adresse aux étudiants du niveau moyen, censés avoir déjà étudié la grammaire de leur langue maternelle. Les élèves apprennent la formation des verbes et les quatre conjugaisons que l'on reconnaît, non par la terminaison de la première personne du présent de l'indicatif, comme en grec, mais par celle de l'infinitif (-er, -ir, -oir ou -re). L'auteur recommande que les débutants écrivent les verbes dans leur cahier, car l'écriture enseigne l'orthographe et renforce la mémoire. Il conseille apprendre d'abord à écrire les tableaux des verbes imprimés dans le manuel et ensuite à les réciter par cœur.

Les pages 62-176 contiennent des morceaux choisis d'auteurs connus, principalement du XVII et $\mathrm{XVIII}^{\mathrm{e}}$ siècles ${ }^{14}$, qu'il faut enseigner selon la méthode de "rumination " ( $\alpha v \alpha \mu \alpha ́ \sigma \eta \emptyset \sigma \varsigma)$ de Jacotot. Herculides sélectionna des extraits qui, à son avis, pouvaient non seulement intéresser les élèves mais aussi leur inspirer "quelque chose d'utile ", les encourager à lire, plus tard, les ouvrages en entier et leur apprendre à rédiger avec éloquence un récit, une description, une allégorie, une pensée philosophique, etc. À la fin (p. 177-180) il explique les parties déclinables du discours (articles, adjectifs, pronoms) et traduit en grec les mots français cités dans le texte (p. 181-222).

On aurait aimé avoir un peu plus d'informations sur la manière dont Herculides appliquait la méthode de Jacotot dans ses cours. Celles qu'il donne concernent la prononciation, la conjugaison des verbes et le vocabulaire. Pour enseigner la prononciation, il conseille de référer les élèves à la prononciation des lettres de l'alphabet de leur langue maternelle. Là où il n'existe pas de phonème identique ${ }^{15}$, le maitre articule le son de vive voix et demande aux élèves de l'imiter.

En morphologie, son attention se concentre principalement sur les verbes. Il recommande que l'apprentissage de leurs formes avance de pair avec l'explication des textes. Il affirme aussi que celui qui connaît les formes des verbes réguliers apprendra facilement à conjuguer les verbes irréguliers. Enfin, il conseille aux maîtres d'exercer les élèves à trouver seuls les temps dérivés à partir des temps primaires.

Quant au lexique, il souligne que la connaissance du sens primitif grec des mots français figurant dans les textes étudiés ne suffit qu'aux débutants. Les élèves avancés doivent apprendre aussi les nuances des mots, en particulier des vocables grecs adoptés par le français, tels que : diplomatique, pathétique, phénomène, etc., car ils n'ont pas le même sens dans les deux langues.

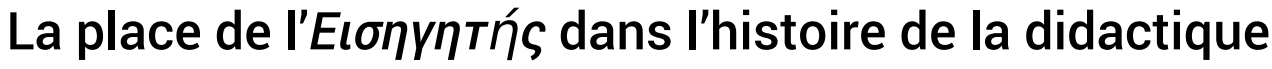 des langues en Grèce}

Le manuel d'Herculides n'apporte rien de particulièrement original à la didactique des langues. Élisabeth Papageorgiou-Provata (1994: 55) affirme que l'auteur prit pour modèle le manuel de A. Lemonnier : Nouvelles leçons de littérature et de morale ${ }^{16}$. Cela n'est pas tellement important : il est connu que la plupart des auteurs des manuels de langue "picorent" dans les ouvrages de leurs prédécesseurs et parfois les copient sans vergogne. L'érudit éditeur Andréas Koromilas ${ }^{17}$ reconnaît qu'Herculides a fait avec «ce premier manuel de français " une contribution considérable à l'avancement des études françaises en Grèce. Il rappelle cependant que « l'Eıøn- 


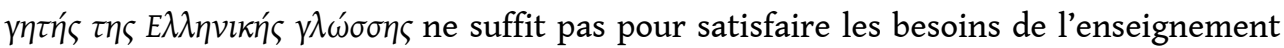

de cette langue» (Papageorgiou-Provata 1994: 55). Enizrexa $r_{70} \cdot 1136^{\circ}$

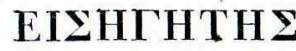

THE

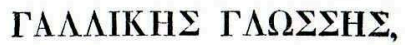
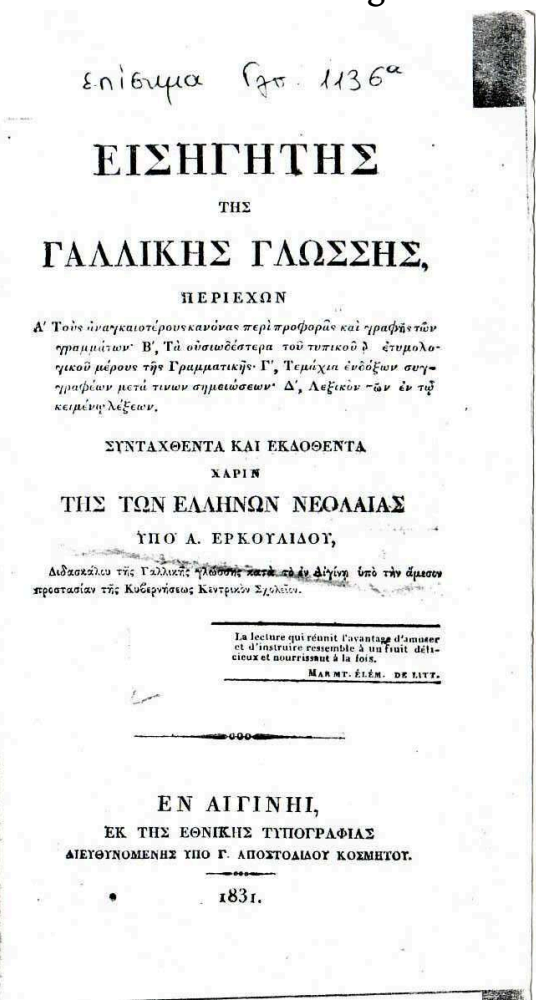

Tel n'était pas, comme il a été déjà dit, le but de l'auteur. En effet, le manuel d'Herculides vise les élèves avancés. Il n'enseigne pas les rudiments de la langue, que les élèves sont supposés avoir déjà maîtrisés, mais la langue française à partir de textes littéraires et cela selon la "méthode universelle» de Jacotot. Il ne se limite pas toutefois, comme Jacotot, à la lecture de Télémaque de Fénelon mais préfère exhiber sa richesse et sa beauté dans une variété de styles. Dans ce sens son livre comblait un vide et fut pour cela largement utilisé, en dépit des terribles accusations de Coray contre son auteur.

\section{La tache indélébile de Coray}

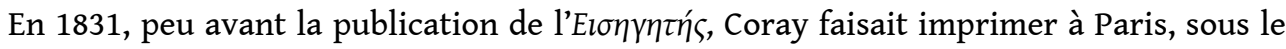
pseudonyme Pantazides, le premier tome d'un ouvrage peu connu, intitulé $\Sigma \hat{u} \mu \mu \imath \kappa \tau \alpha$ $\varepsilon \lambda \lambda \eta v i k \alpha ́$ [Mélanges grecs] (Valetas $1964: 673-733)$, un recueil d'extraits de « dépêches » qu'il prétendait recevoir de compatriotes "fiables vivant en Grèce ", qu'il ne nomme pas) ${ }^{18}$. Les $\Sigma u ́ \mu \mu \imath \kappa \tau \alpha$ commencent par le «rapport» (ibid.: 675-683) d'un auteur anonyme, envoyé d'Égine le 15 décembre 1830 à Pantazides (Coray), à Paris. L'auteur du document analyse la politique "obscurantiste» de Capodistrias dans le domaine de l'éducation et commente la crise à l'École centrale à Égine, provoquée par les « manigances» du Gouverneur et de ses amis contre Venthylos.

Lorsque ce dernier soumit, en signe de protestation, sa démission, l'administration de l'École nomma pour enseigner le français un certain Herculides, appelé communément "Vulcanides $"^{19}$, à cause de son infirmité. Or, continue l'auteur du rapport anonyme, contrairement à Venthylos, ce monsieur qui avait appris le français en Russie, est 
complètement « ignare et inculte », ignore aussi bien le grec ancien ( $\varepsilon \lambda \lambda \eta v i k \eta ́)$ que le français et traite ses élèves, dont la plupart ont plus de vingt ans, comme des jeunes écoliers. On sait bien, conclut-il, la raison pour laquelle des gens de cette espèce sont nommés à ces postes. Ce qui est cependant étonnant est qu'il existe encore des individus comme lui qui n'hésitent pas d'accepter un travail pour lequel ils n'ont pas été formés! (ibid. : 681).

Je ne sais pas si Herculides était vraiment boiteux, où il avait appris le français, comment il traitait ses élèves ni qui le nomma à l'École centrale et pourquoi. Ce que je sais est que les accusations sur son ignorance et son manque de culture sont injustes,

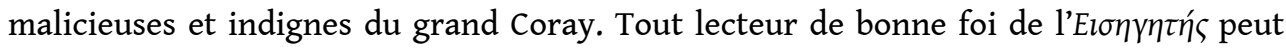
s'en convaincre facilement.

Comment expliquer la conduite odieuse du sage Grec contre un homme qu'il ne connaissait pas, de qui il n'avait jamais entendu parler auparavant et dont il n'avait ni vu ni lu le livre ${ }^{20}$ ? Pour répondre à cette question il faudrait examiner les idées politiques de Coray et étudier l'évolution de ses rapports avec le Gouverneur ${ }^{21}$, ce qui dépasse le cadre de ma communication. Je me limiterai pour cela à une explication sommaire.

24 Coray, témoin oculaire et admirateur de la Révolution française, était un défenseur farouche de la Déclaration des droits de l'homme et du citoyen, un républicain fervent et un intellectuel passionné de justice sociale. Apprenant que Capodistrias dirigeait le pays sans tenir compte de la constitution et sans consulter le peuple ni les chefs de guerre ou les notables, Coray se sentit trahi par lui ${ }^{22}$.

Voyant son rêve d'une Grèce libre, démocratique et républicaine s'évanouir, il jugea de son devoir d'empêcher par tous les moyens, avant de mourir ${ }^{23}$, l'ancien ministre du tsar et ses collaborateurs (ses "satellites » comme il les appelle), d'imposer en Grèce un régime despotique. Et puisque ses informateurs lui indiquaient qu'Herculides en était un, il fallait le neutraliser aussi. Il se sert dans ce but de demi-vérités, de rumeurs, de ragots et de mensonges que lui rapportent les adversaires, comme lui, de Capodistrias, sans jamais se donner la peine de les vérifier. Il se moque du professeur de français, le ridiculise, le vilipende et accuse le Gouverneur, son ancien ami, de vouloir instaurer un régime autoritaire et maintenir le peuple dans les ténèbres pour transformer les Grecs en esclaves ${ }^{24}$. La preuve, affirme-t-il, en est que la situation dans le domaine de l'éducation est depuis son arrivée bien pire qu'elle ne l'était sous l'occupation turque et qu'elle ne s'améliorera pas tant que le « sultan Yannis », comme il l'appelle, occupera le pouvoir!

26 Le 19 octobre 1831, Capodistrias est assassiné et les passions se déchaînent. Il faudra presque trois ans pour que le calme revienne. La responsabilité morale de Coray est incontestable. L'érudit N. Pangalakis, compatriote et ami intime de Coray et de Capodistrias, envoie alors une longue lettre au « sage vieillard de Paris », dans laquelle il lui écrit: "Ton Pantazides [les $\Sigma u ́ \mu \mu \imath \kappa \tau \alpha$, le libelle contre le Gouverneur] est une tache sur ta vie irréprochable, que tu dois laver $»^{25}$ (Daskalakis 1979: 594). Mais Coray ne s'est jamais repenti. Au contraire, il continua d'approuver l'assassinat du Gouverneur jusqu'à la fin de ses jours. "Le tyran a été puni justement (quoique par la main d'un assassin injuste) par Dieu, vengeur de toute injustice ${ }^{26}$ (ibid. : 639). 


\section{La réaction d'Herculides}

27 Le 3 juin 1832, Herculides, en apprenant les médisances que Pantazides (Coray) répandait sur son compte, lui fit parvenir un exemplaire de son manuel, accompagné d'une lettre en français, dans laquelle il protestait avec force contre les allégations mensongères publiées ${ }^{27}$ dans les Mélanges grecs. Coray, surpris et fort embarrassé, tenta

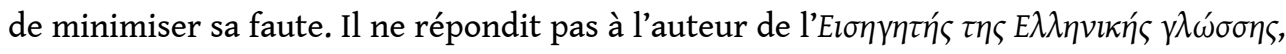
mais le 18 août 1832, en informa par écrit ses soi-disant «amis éditeurs des $\Sigma u ́ \mu \mu \imath \kappa \tau \alpha$ $\varepsilon \lambda \lambda \eta v \iota k \alpha ́$ » à Paris, Fournarakis et Rallis, deux jeunes Chiotes qui appartenaient à ses partisans les plus dévoués. Dans cette lettre, Pantazides (Coray) reconnaissait qu'Herculides n'est pas inculte et il se défend d'avoir jamais mis en cause ses mœurs et sa conduite. Seule son instruction fut mise, dit-il, en doute, et cela non par Pantazides mais par l'« auteur » (anonyme) de la dépêche du 15 décembre 1830.

Ce dernier, écrit Coray, trompé par ce nom inusité, Herculides, le prit pour un étranger, donc pour quelqu'un qui n'avait pas fréquenté l'école grecque et par conséquent ignorait les finesses de la langue et de la culture grecques. Pantazides proclame par la suite qu'il ne croit pas personnellement qu'Herculides soit ignorant et sans culture.

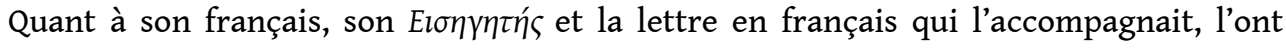
persuadé, dit-il, que non seulement il a une solide connaissance de cet idiome mais bien d'autres connaissances aussi, en particulier sur l'art d'enseigner la langue française aux jeunes. Si, néanmoins, ses élèves à l'École centrale d'Égine ne profitèrent pas suffisamment de ses cours ni de ses efforts inlassables pour leur être utile, la faute en incombe au gouvernement de Grèce de l'époque, qui n'avait pas cessé de harceler le professeur...

29 Coray n'exprima aucun regret pour les mensonges et les calomnies publiées dans $\Sigma u ́ \mu \mu \imath \kappa \tau \alpha$ et ne s'en excusa pas. Au contraire, il conseilla à Herculides de se sentir fier des humiliations que lui fit subir le «sultan Yannis » (comme il appelle le Gouverneur Ioannis Capodistrias (ibid.: 683). "Quelle autre plus brillante preuve de son éducation et de sa conduite morale pouvait souhaiter Herculides, que la haine du despote de la Grèce? » (ibid. : 650).

30 Je ne sais pas si l'auteur du premier manuel de français publié en Grèce fut jamais informé du contenu de cette lettre de Coray. De toute façon, cela ne changerait rien. Le mal était fait, d'autre part les événements se succèdent rapidement. En 1833, Coray mourait à Paris; la même année la Grèce devenait une monarchie héréditaire et en 1834 l'École centrale d'Égine fermait ses portes définitivement et on perd alors les traces d'Herculides. On le retrouve en 1838, à la veille de sa nomination comme

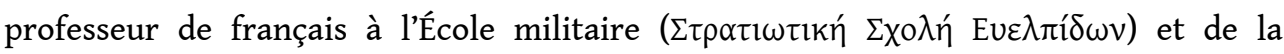

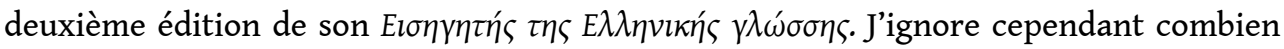
de temps il professa à cette école et avec quel succès et où et comment il finit ses jours.

\section{Conclusion}

31 Herculides n'est certes pas Coménius ni même Lhomond. Son nom est inconnu en dehors de la Grèce. Même dans sa patrie, seuls les spécialistes se souviennent encore de lui. Pourtant il joua un rôle important dans l'histoire de l'enseignement des langues modernes en Grèce et donc, dans la diffusion de la langue française dans le bassin 
méditerranéen. Herculides était un maître des langues compétent, un didacticien bien informé et un auteur méthodique, clair et précis. Des milliers de jeunes grecs apprirent

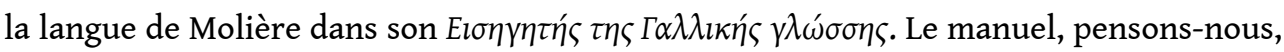
et son auteur méritent bien une place dans l'histoire de la didactique des langues. De même que les guerres ne se gagnent pas seulement par les généraux, l'histoire de la didactique des langues ne s'écrit pas seulement par les génies. D’ailleurs les génies sont

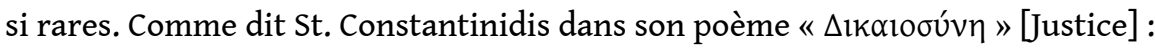

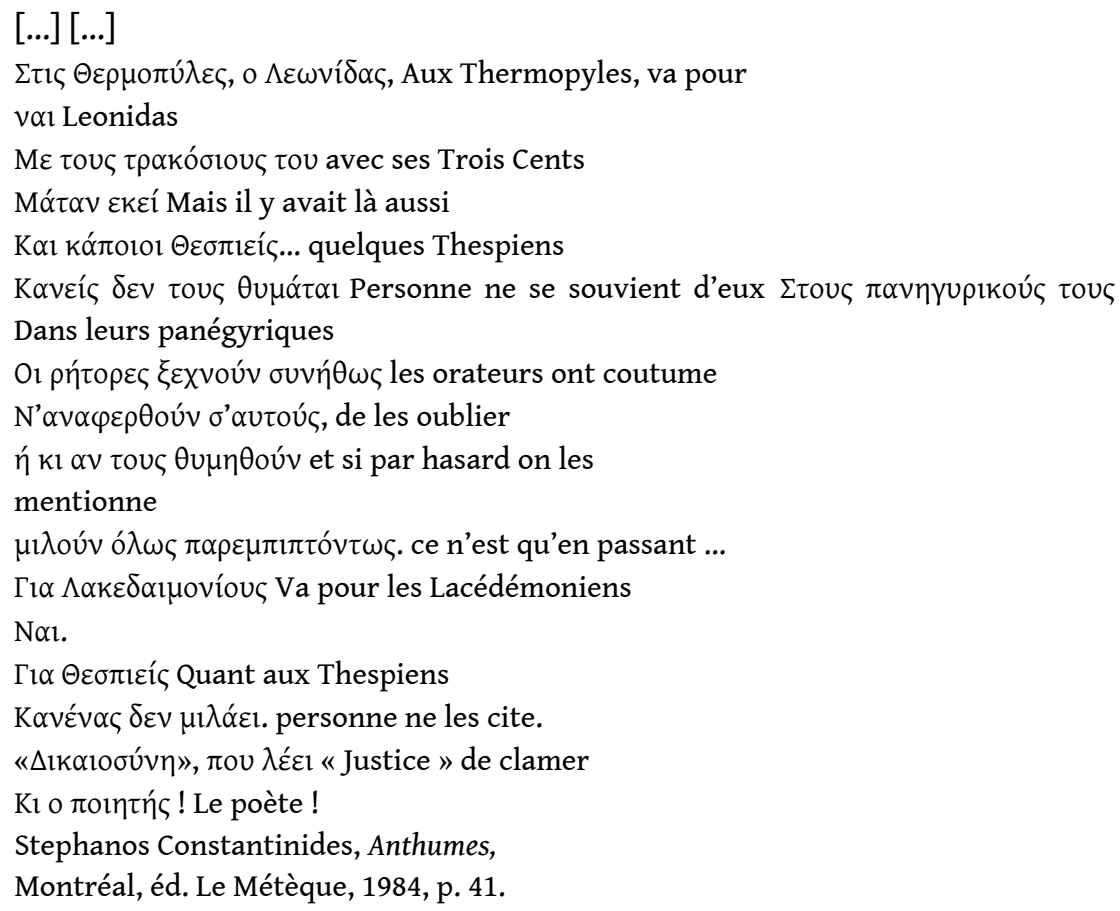

\section{BIBLIOGRAPHIE}

CARAVOLAS, Jean-Antoine (2000). Histoire de la didactique des langues au siècle des Lumières. Précis et anthologie thématique. Montréal, Tübingen : Les Presses de l'université de Montréal - Gunter Narr Verlag.

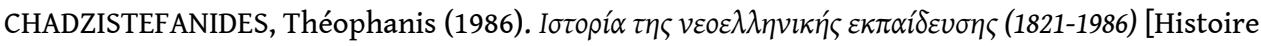
de l'éducation grecque (1821-1986)]. Athènes : Papadimas.

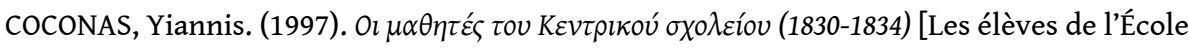
centrale (1830-1834)]. Athènes : Archives historiques de la jeunesse hellénique - Centre des études néohelléniques - Fondation Nationale de la Recherche Scientifique.

CORAY, Adamance (1799). Les caractères de Théophraste. Traduction française de A. Coray. Paris : Eberhard.

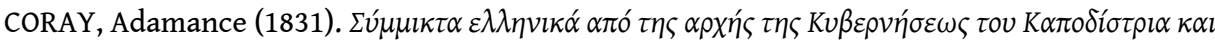
$\varepsilon \varphi \varepsilon \xi \eta ́$ ऽ [Mélanges grecs depuis le début du gouvernement de Capodistrias]. Paris : Eberhard. 


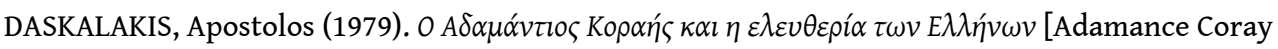
et la liberté des Grecs]. Athènes : E. G. Vayionakis.

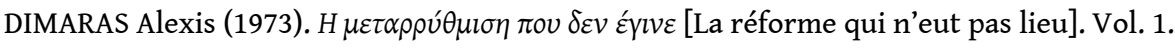
(1821-1894) \& vol.2. (1895-1967). Athènes : Hermès.

FRANGHISCOS, Emmanuel N. (2005). « A survey of Studies on Adamantios Korais during the Nineteenth Century ». The Historical Review. Institute for Neohellenic Research. National Hellenic Research Foundation, Vol. 2 : 93-128.

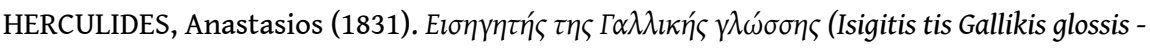
Introduction à la langue française. Égine : Ethniki typografia.

LEMONNIER, A. (1822). Nouvelles leçons de littérature et de morale, Paris,

NICOLAÏDIS, Dimitri (1992). D’une Grèce à l'autre. Paris : Les elles lettres.

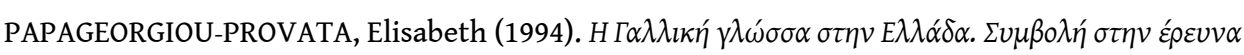

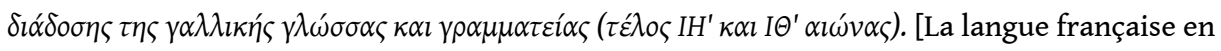
Grèce. Contribution à l'étude de la diffusion de la langue et de la littérature françaises]. Athènes : Fondation des études néohelléniques.

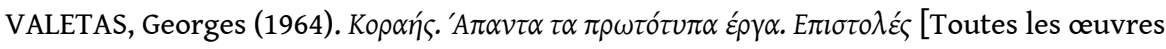
originales. Lettres]. Vol. B.1 (1774-1814), Vol. B.2. (1815-1833) Athènes : Dorikos.

\section{NOTES}

2. Le nombre d'orphelins était énorme à la fin de la guerre de l'indépendance.

3. Formée avec beaucoup de difficulté par Charles-Nicolas Fabvier (1782-1855), colonel napoléonien, libéral et philhellène, elle était à la libération en pleine décomposition. Au début de 1829, à la tête de l'armée régulière est nommé le philhellène bavarois colonel Heydek, mais il l'équipe d'armes françaises, et confie la direction de l'École militaire au lieutenant-colonel Pauzier, commandant de l'artillerie grecque. En mai 1829, des dizaines d'officiers français qui sous le commandement du général Nicolas Joseph Maison (1771-1840), avaient participé à l'expédition française de Morée (1828-1829), entrent dans l'armée grecque; quarante sousofficiers sont promus sous-lieutenants pour servir d'instructeurs de l'armée grecque; les soldats grecs portent l'uniforme bleu français. Le colonel Pellion, est nommé commandant de la cavalerie, le lieutenant-colonel Saint Martin commandant de l'Intendance et le colonel Garneau commandant des unités de fortifications et de mécaniciens. Après la démission de Heydek pour des raisons de santé, le lieutenant général Camille Trézel (1780-1860), autre membre de l'expédition de Morée, est nommé commandant général des forces armées grecques régulières. En août 1830 il est remplacé par le général Gérard, l'armée grecque est dissoute et les officiers français partent. Seuls deux bataillons français, sous le commandement du général Guénou, restent et tentent avec une unité anglaise de maintenir l'ordre.

4. Colettis, médecin, participa à la guerre de l'indépendance et joua un rôle de premier plan dans la vie politique de la Grèce (il fonda le parti français, fut ambassadeur à Paris de 1835 à 1843, plusieurs fois ministre et premier ministre).

5. Venthylos passa alors au gymnase de Nauplie et en 1837 à l'Université d'Athènes où il enseigna l'ancien grec jusqu'à sa mort (Papageorgiou-Provata 1994 : 43).

6. Selon le rapport de la police le professeur de grec ancien, géographie et histoire Gennadios incitait les élèves contre le Gouverneur et son gouvernement et contre Herculides, qu'il 
considérait loyal au Gouverneur (Alexis Dimaras 1973: 32). Selon J. Coconas (1997: 60-65) Herculides signa la pétition progouvernementale d'août 1832 .

7. Certains, toutefois, quittent l'école après avoir publié, le 1er février, une proclamation où ils affirment avoir été forcés de se repentir ou aller en prison ou en exil et où ils protestent violemment contre les mesures répressives de Capodistrias

8. Le 31.12.1836 - table des matières : français et latin. À l'École grecque (Hellinikon scholeion-3 ans) - Français : 4 heures par semaine ; Latin : 3e année - 3h. par semaine. Au Gymnase (4 ans) : Français : 2 heures par semaine ; Latin : $4 \mathrm{~h}+6+5+4$. Total : Français 7 ans $20 \mathrm{~h}$; Latin 5 ans $-22 \mathrm{~h}$. (Dimaras $1973: 66$ ).

9. L'ouvrage était destiné aux élèves qui étudiaient le français avec lui à l'École centrale d'Égine.

10. Se référant aux Leçons préliminaires de Condillac il répète qu'il est impossible de décrire comment la méthode d'enseignement est appliquée dans la salle de classe et que pour le savoir il faut plutôt consulter le cahier de l'élève qui est le « miroir » de la leçon.

11. Il la décrit brièvement dans une notice de bas de page. Elle consiste, écrit-il, à présenter aux jeunes les écrits d'un nombre restreint d'excellents auteurs. Les élèves répètent (« ruminent») les textes jusqu'à ce que ceux-ci se transforment en «bouillie » qui leur servira d'aliment de l'esprit et de fondement de la philologie.

12. Par exemple : «Quelqu'un ayant fait mettre sur la porte de sa maison cette inscription : Que rien de mauvais n'entre2 par ici, Diogène demanda3 par où donc entrera le maître »4. Note : Les mots numérotés sont expliqués en grec au bas du texte.

13. a) Entre le père et le fils sur l'obligation des enfants à obéir à leurs parents (p. 19-27) ; b) De la

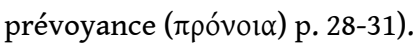

14. On y trouve des morceaux choisis de d'Aguesseau, de l'abbé Barthélémy, de Bernardin de Saint-Pierre, de Choiseul-Gouffier, Chateaubriand, Fénelon, Marmontel, Montesquieu, Rousseau, Volney, mais aussi de Bossuet et de La Bruyère, et également du poète anglais Addison. Étrangement, Voltaire en est absent.

15. Ces mots sont marqués d'un astérisque.

16. Ce livre est rare. Je n'ai pas encore réussi à le localiser.

17. Koromilas (1811-1858), originaire d'Athènes, participa très jeune à la guerre d'indépendance et fut blessé. Envoyé à Paris, il apprit chez Ambroise Firmin Didot l'art de l'imprimerie. À son retour en Grèce, il fonda, en 1830, une imprimerie à Égine et publia entre autres les ouvrages de son protecteur N. Vamvas (1770-1856), le précepteur de la nation. En 1836 il transféra son imprimerie (la plus importante maison d'édition du Moyen Orient) à Athènes. La plupart des futurs imprimeurs-éditeurs grecs apprirent le métier chez lui.

18. La distribution de l'ouvrage en Grèce et partout où vivait et travaillait la diaspora grecque, était assurée par les nombreux amis et admirateurs de Coray.

19. Allusion à Vulcain, nom latin de Héphaïstos, dieu du feu et des métaux, qui comme on sait était boiteux.

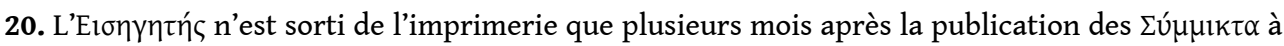
Paris.

21. On trouvera sur ces deux sujets de nombreuses interprétations dans les innombrables publications sur Coray (voir, entre autres, l'article de Frangiskos 2005 : 93-128).

22. Les deux hommes se connaissaient depuis longtemps, s'étaient rencontrés plusieurs fois à Paris et éprouvaient l'un pour l'autre la plus grande admiration et le plus profond respect Le 26 décembre 1815, Coray écrivait à Vienne à Alexandre Vasileiou, son ami le plus proche : «J'ai connu Capo d'Istria et cela avec grand plaisir» (Valetas 1964, t. 2, vol.2: 52). Le 22 septembre 1818, il écrivait à un autre ami grec : « Ne prends pas pour flatterie quand je le désigne 'orgueil de

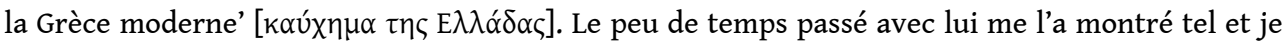
ne crois pas qu'il m'ait trompé » (ibid. : 168). Il salua son élection au poste de Gouverneur avec 
enthousiasme et encore le 2 mai 1830 il lui écrivait : " Je loue ce que tu as fait pour l'instruction du peuple » (ibid. : 599).

23. Coray avait alors plus de quatre-vingts ans.

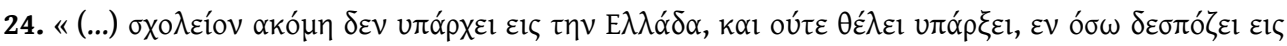

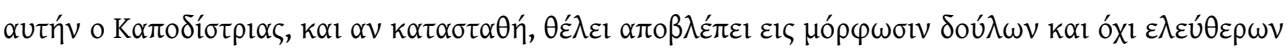

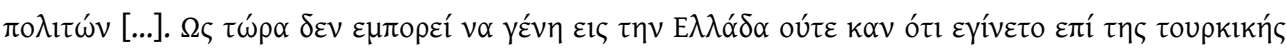

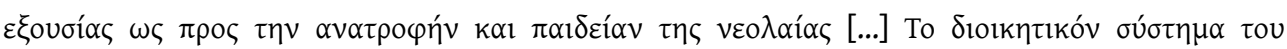

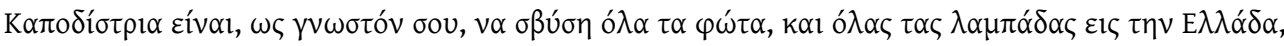

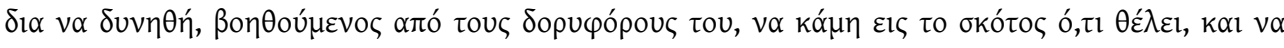

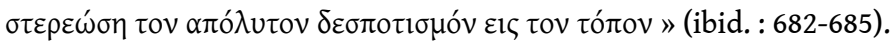

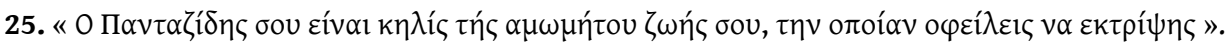

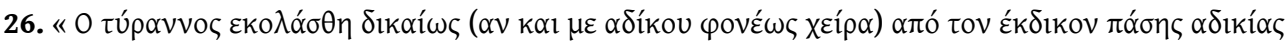
ఆróv [...]».

27. Coray n'a pas publié cette lettre de Herculides. Nous ne savons de son contenu que ce qu'il écrit dans sa lettre à Fournarakis et Rallis le 18 août 1832 (Valetas 1964, t. 2. vol. 2 : 649-650).

\section{RÉSUMÉS}

L'enseignement méthodique du français en Grèce moderne commence à l'École centrale d'Égine, en 1829, avec l'arrivée d'Anastasios Herculides. Accusé injustement d'être ignorant, incompétent et l'instrument aveugle du Gouverneur Ioannis Capodistrias (1776-1831), les étudiants, encouragés surtout par A. Coray, se révoltent et demandent le départ de tous les deux. Le

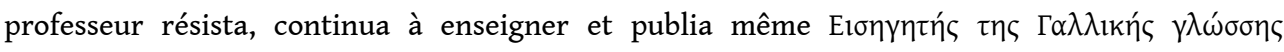
(Introduction à la langue française), le premier manuel de français pour Grecs, imprimé en Grèce après l'indépendance. Le Gouverneur fut pendant les désordres assassiné. En 1838, on retrouve

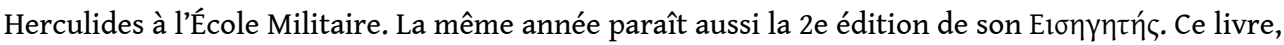
inspiré de la Méthode universelle de Jacotot, s'adresse principalement aux élèves du niveau moyen et avancé. Il s'agit d'un ouvrage bien structuré qui enseigne à parler et à écrire le français par la lecture de textes littéraires d'auteurs variés, pas seulement de Télémaque comme le fait Jacotot. Même Coray dut, avant de mourir, admettre que l'auteur avait une solide connaissance $\mathrm{du}$ français et qu'il maitrisait bien l'art d'enseigner la langue aux jeunes. Néanmoins il ne s'est jamais repenti du mal qu'il causa ni au Gouverneur Capodistrias, son ancien ami, ni à Herculides, un des pionniers de l'enseignement méthodique du français langue étrangère en Grèce.

The teaching of French in the schools of Modern Greece begins in 1829 at the Central School of Aegina, after the arrival there of Anastasios Herculides. Based on unfair accusations by A. Coray and others, of being an obedient instrument of the Governor of the country, Ioannis Capodistrias (1776-1831), as well as of ignorance and incompetence, the students of the school revolted and demanded the departure of both of them. The teacher resisted, continued his classes and, in

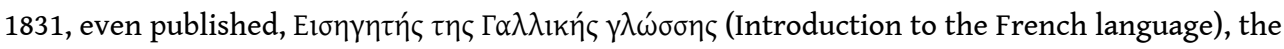
first French textbook for Greeks published in independent Greece. The Governor, later the same year, was shot and killed. In 1838, we find Herculides teaching French at the Military School. The

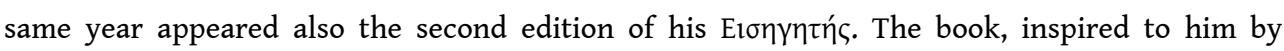
Jacotot's Méthode Universelle, is written mainly for middle and advanced level students. It is a 
well structured book, teaching to speak and write the French language through the reading of literary texts by a variety of authors, not only Fenelon's Télémaque, as Jacotot does. Even Coray

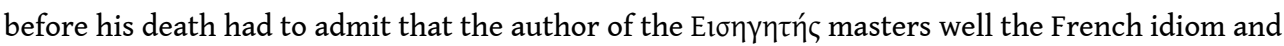
is knowledgeable of many things, notably the art of teaching the French language to the young ! Nevertheless, he never apologized for the harm he caused to his former friend, the Governor I. Capodistrias nor to Herculides, one of the pioneers of methodical teaching of the French language in modern Greece

INDEX

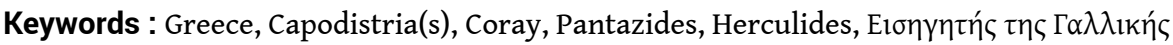
$\gamma \lambda \omega ́ \sigma \sigma \eta \varsigma$ (Introduction à la langue française), Jacotot, méthode universelle, Central School of Aegina, students rebellion 1831

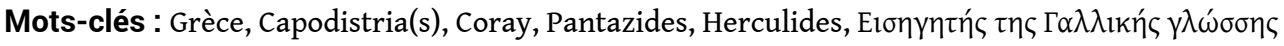
(Introduction à la langue française), Jacotot, méthode universelle, École centrale d'Égine, révolte des étudiants 1831

\section{AUTEUR \\ JEAN ANTOINE CARAVOLAS}

Montréal 